\title{
Improving access with a collaborative approach to cancer genetic counseling services: a pilot study
}

\author{
Stephanie A. Cohen, MS, CGC, LGC, and Dawn E. McIlvried, MS, CGC, LGC \\ St. Vincent Hospital Center for Cancer Care, Cancer Genetics Risk Assessment Program, Indianapolis, Indiana
}

Background Limited access to cancer genetic counselors (GC) may result in the lack of patient identification and/or failure to show due to travel distance and complicated treatment schedules.

Objective We hypothesized that access would improve when a GC collaborated with distant nongenetics health care providers to provide services locally.

Methods Patients at a collaborative site were offered a risk assessment survey that was reviewed remotely by a licensed, boardcertified GC. Patients were triaged such that the onsite registered nurse (RN) provided basic risk assessment and offered genetic testing for straight-forward hereditary breast and ovarian cases. Ongoing training and support was provided by the GC. Followup and complex cases were scheduled with the GC during a monthly outreach visit to the collaborative site.

Results During the 1-year study period, the total number of patients who accessed genetic counseling services from the target region was 4 times greater than the previous year. Ten of 17 patients who were triaged for genetic counseling and testing underwent genetic risk assessment services as a result of this identification and triage protocol.

Conclusion This defines a workable approach for patient identification and triage for hereditary cancer risk assessment and genetic counseling in a community setting. This collaborative approach may be applicable to centers that do not have access to a board-certified GC, especially important in light of the 2012 Commission on Cancer Standards that require cancer risk assessment, genetic counseling and testing services on site or by referral.

\begin{abstract}
$\longrightarrow$ ancer genetic counseling is a process that involves collecting and interpreting detailed medical and family history, assessing risk for hereditary cancer, offering appropriate genetic testing and providing follow-up. Hereditary cancer comprises approximately 5\%-10\% of cancers in general. Identifying at-risk individuals is important to guide clinicians in surveillance and surgical decision making, as well as medical management plans following a cancer diagnosis. Additionally, family members may be at high risk for certain cancers, and may need enhanced surveillance to identify cancer at an earlier and more treatable stage.

In recent years, there has been an increasing demand for genetic testing for hereditary cancers to assist in cancer surveillance and treatment planning. ${ }^{1,2}$ There are 2,700 board-certified genetic

\footnotetext{
Manuscript received December 12, 2012; accepted May 6, 2013. Correspondence Stephanie A. Cohen, MS, CGC, LGC, St. Vincent Hospital Center for Cancer Care, Cancer Genetics Risk Assessment Program, 8301 Harcourt Road, \#100, Indianapolis, IN 46260 (sacohen@stvincent.org).

Disclosures Ms. Cohen has been a paid speaker and served on an advisory board for Myriad Genetics Laboratories.
}

counselors, with approximately 675 who specialize in hereditary cancer, according to the National Society of Genetics Counselors 2012 Professional Status Survey. As a result, more nongenetics trained clinicians are ordering genetic tests. Although primary care physicians acknowledge that they play a role in cancer genetic services, they feel unprepared to address genetic testing issues. ${ }^{3-8}$ Additionally, there is a perceived lack of access to genetic services. ${ }^{9}$

Several organizations have recognized the importance of a trained genetics professional in the process of hereditary cancer risk assessment, and have called for testing to be conducted with the involvement of a health care professional with genetics training, such as a board-certified genetic counselor. $^{10-12}$ In fact, the 2012 Commission on Cancer Standards dictate that genetic risk assessment be provided by a qualified genetics professional. ${ }^{13}$ At the time this project was taken on, the state of Indiana had 4 board-certified genetic counselors dedicated to cancer genetics, 3 of DOI: 10.12788/j.cmonc.0031 
whom were centrally located in Indianapolis. This number has risen to 6 over the past 2 years, but still leaves a large portion of the state without geographic access to a specialized provider. This is not unlike many other states in the country, where services tend to be concentrated in major medical centers in large cities.

Training of nurses to provide education about breast cancer susceptibility genetic testing appears to result in no difference among patients in understanding, satisfaction or perceived autonomy in the test decision making process compared to genetics professionals. ${ }^{14}$ An important component of this process is the ability of nongenetics health care providers to recognize their limitations and knowing when to refer cases to a genetics professional specializing in cancer. A collaborative approach to providing cancer genetic services has been described as a way to use local staff to address straightforward cases, while triaging more complex cases to a trained cancer genetics provider. ${ }^{15}$ This triage system allows for provision of services locally for those individuals who do not want (or are not able) to travel a long distance for genetic risk assessment. Since it is a collaborative relationship, patients and local providers have access to board-certified genetic counselors as needed, and the genetic counselor's time and resources are focused on the most complex cases.

Cancer Center staff from a small 167-bed hospital contacted the St. Vincent Cancer Genetics Risk Assessment Program in Indianapolis to provide genetic services at their facility. The smaller facility, located about 60 miles away, had 211 new cancer diagnoses (all cancer types) in 2010. Considering that roughly $5 \%$ of all cancers are due to a hereditary cause, this would translate into as many as 42 oncology patients in 2010 who may have been appropriate for risk assessment. The cancer genetics program staff evaluated the remote hospital's needs and the time and services that could be provided by current staff in both locations. The staff had received some minimal training from a genetic testing company and ordered genetic testing for a few patients, but did not feel confident in their ability to provide risk assessment or comfortable with the testing process. A major contributing factor in staff reluctance to offer genetic testing was that an apparently straightforward case may be more complicated than initially anticipated. This situation leads to potential liability for ordering incorrect testing and missing differential diagnoses. These problems are consistent with literature that reports discomfort by nongenetics health care providers in providing genetic services. ${ }^{3-8,12}$

Patients from the remote site were occasionally being referred to the St. Vincent Cancer Genetics Risk Assessment Program in Indianapolis, but not with the consis- tency or frequency that would be expected for a hospital with their patient population. Additionally, upon review of data from the cancer genetics risk assessment program, many of the referred patients were failing to schedule or keep their appointments. Reasons for patient failure include being overwhelmed with multiple appointments, distance to travel, the time necessary for an appointment and concern about insurance coverage. We hypothesized that some of these issues may be alleviated by offering local cancer genetic risk assessment within a patient's community to allow increased access to a service that might otherwise not be used.

We proposed that a collaborative approach to hereditary cancer services would be beneficial for the remote site's patient and physician community as well as the St. Vincent Cancer Genetics Risk Assessment Program. Patients identified at high risk for cancer by gene testing or family history would have different surveillance recommendations and management options that may allow for early detection of cancer or a reduction in cancer risk. Patients would have the advantage of being served within their local community, where they already receive treatment and are familiar with the facility and providers. If an individual was identified at increased risk for cancer and therefore required additional surveillance, they would likely seek those services locally, if possible. This approach would allow the remote site to be affiliated with boardcertified genetic counselors, and thus able to offer a higher level service to their patients. Better knowledge and increased confidence in providing genetic testing by local staff at the remote site would improve the quality of patient care. The assessment program would also benefit from increased patient volume by identifying those who need to see a board-certified genetic counselor.

In light of these issues regarding adequate access to cancer genetic counselors in our region, this study set forth to pilot a collaborative model of providing genetic services. We hypothesized that collaboration of boardcertified genetic counselors with nongenetics health care providers to handle basic genetic testing at a local facility is a feasible approach to provide genetic services and would improve access to cancer genetic services. We anticipated that local, nongenetics health care providers' knowledge and confidence regarding cancer genetics and genetic testing issues would improve with specialized training by and collaboration with board-certified genetic counselors.

\section{Methods}

A collaborative approach was adopted to provide local cancer genetic services. This approach included identifying a key person at the collaborative site; in this case, a 
registered nurse $(\mathrm{RN})$ employed by the collaborative site's cancer center, who would provide genetic evaluation/testing services. We also identified a supporting physician (MD) at the collaborative site who would be able to order tests and oversee the RN.

Training of the nongenetics staff at the collaborative site included completion of the Oncology Nurses Society (ONS) on-line course on cancer genetics by both the RN and MD. The RN observed patient sessions with a board certified genetic counselor at St. Vincent Hospital and patient sessions with a nurse practitioner in the St. Vincent Breast Risk Assessment Clinic. The RN and MD were provided with reading materials on the subject of cancer genetics. The collaborative site staff was given exam questions following the ONS course completion. They re-took the same exam after 1 year following implementation of the collaborative care program to assess their knowledge after several months of application. The goal of this training was to increase confidence in offering genetic testing, while recognizing limitations in cases that were nonroutine and/or with unexpected test results.

The collaborating site was provided with a tablet computer to collect initial family history information for the purposes of cancer risk assessment. The $\mathrm{RN}$ offered $\mathrm{pa}^{-}$ tients being treated at the remote site in radiation oncology or medical oncology the opportunity to complete the computerized family history survey at the time of their appointment. Some amount of prescreening was done, so if the patient did not have any family history of cancer and/or was not young at the age of diagnosis, the survey was not offered. Use of a shared network allowed the genetic counselors (GC) remote access to family history information to triage cases. The St. Vincent site provided technical support and training to use the computer tablets. Ongoing genetic counseling support was provided throughout the process in a collaborative manner.

The GCs reviewed the information generated from the computer tablet family history collection (Hughes RiskApps program) and triaged cases to be handled locally at the collaborative site or to be seen by the assessment program staff. Each patient was assigned a risk category: "increased risk for hereditary breast and ovarian cancer (HBOC)", "increased risk for hereditary cancer" or "no increased risk for hereditary cancer". These were determined by following published criteria for $\mathrm{HBOC}$ risk identification and professional opinion. ${ }^{16,17}$ A GC traveled to the collaborating site once per month to see patients and attend tumor board to provide input on cases and gain visibility among medical staff. The GC reviewed cases with the $\mathrm{RN}$ at the monthly visits, and were available offsite by email and phone consultation as needed.
Patients who were assigned a risk category of "increased risk for HBOC" were contacted by the local RN who offered to meet with the patient at the time of their next appointment in radiation oncology or medical oncology. At that time, she discussed the option of genetic testing for HBOC, obtained informed consent and collected a blood sample to be sent for BRCA1/2 testing. When results were received, the $\mathrm{RN}$ contacted both the $\mathrm{GC}$ and the patient. The patient was offered a follow-up appointment with the $\mathrm{GC}$ at the collaborating site for evaluation of other hereditary or familial causes for breast and/or ovarian cancer and arrangement of further testing, if necessary. All variants of uncertain significance and positive results were to be seen by the GC, and individuals with negative test results were encouraged to attend a follow-up appointment with the GC.

Patients who were assigned a risk category of" increased risk for hereditary cancer" were contacted by the local RN and offered an appointment with the GC either locally at the collaborative site, or referred to the main cancer genetics program site. Patients who were assigned the "no increased risk for hereditary cancer" category were informed by the RN at their next appointment that they were not a candidate for further risk assessment and genetic testing.

The St. Vincent program was able to obtain grant funding for the collaborative care project for a year. Over the course of this year, the number of high-risk patients identified and seen at the collaborative site was tracked, as well as the number of patients triaged to the St. Vincent program in Indianapolis. For each patient seen either at St. Vincent Indianapolis or at the collaborative site, documentation of genetic testing and the outcome of testing was maintained in an excel spreadsheet. Descriptive statistical analysis was used to tabulate the number of $\mathrm{pa}^{-}$ tients accessing genetic services locally at the collaborative site with the RN versus board-certified GC. Over the course of the year, the time spent on collaborative activities was tracked, including initial educational training, ongoing support, computer intake survey review and $\mathrm{pa}^{-}$ tient triage, travel, attendance at tumor board, and inperson patient consultation.

\section{Results}

We piloted this collaborative process on a total of 17 patients at the collaborative site. Individuals completed a computerized family history survey that was then evaluated and triaged by the GC. Thirteen patients were identified at increased risk; 8 were assigned to the "increased risk for HBOC" category and 5 were assigned to the "increased risk for hereditary cancer" category (Figure 1). Five patients accepted evaluation by the $\mathrm{RN}$ and had 


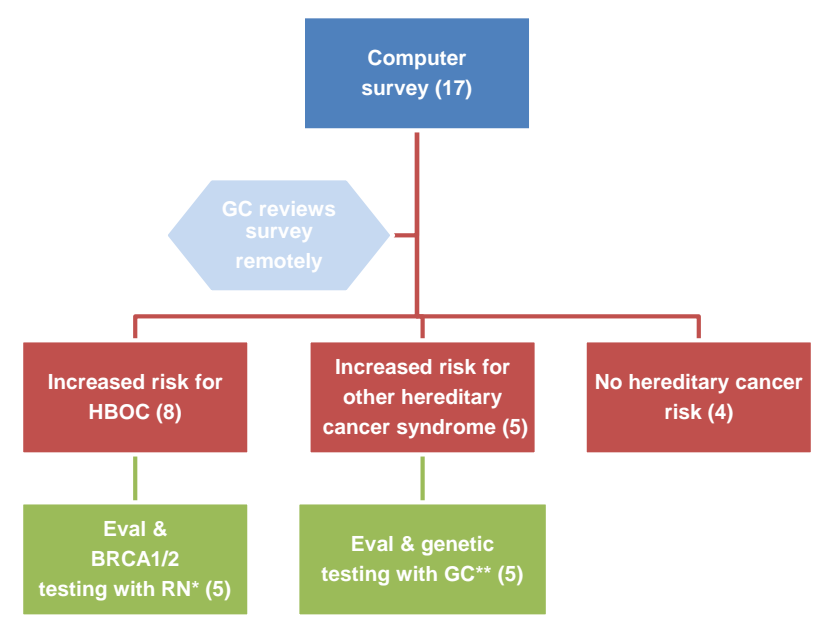

FIGURE 1 Collaborative approach to hereditary cancer services. *GC available as needed and for follow-up results sessions, ongoing joint case reviews. ${ }^{*}$ One patient at general population risk requested consult.

genetic testing for $B R C A 1 / 2$, and 3 declined evaluation. Four out of the 5 patients who underwent testing were seen by the GC in follow-up. Three of these individuals were offered further testing, based on risk assessment by the GC.

Only one patient assigned to the "increased risk for hereditary cancer" category declined evaluation with a GC. Of the 4 who received genetic counseling at the collaborative site, all had genetic testing. One patient assigned to the "no increased risk for hereditary cancer" category requested a consultation with the GC; no testing was performed in this case. In all, 10 patients underwent genetic risk assessment services as a result of this identification and triage protocol.

A review of the cancer genetics database was performed, searching for patients with an address within the city of the collaborative site. A total of 15 patients for the collaborative site city received genetic counseling in Indianapolis from July 2006 through end of June 2009, prior to initiation of the collaborative genetic counseling program (Figure 2). A total of 17 patients with an address of the collaborative site city accessed genetic counseling from October of 2009 through the end of September 2010, either at the main site in Indianapolis or the collaborative site.

Each health care participant (GC, RN, and MD) was asked to track time spent on collaborative activities (Table 1). There were 2 RNs who completed training and were involved with initiation of the collaborative process, but 1 left her position 2 months into the implementation period. She had tracked her time spent on training and educational activities, and this data was used because the second RN did not provide this information. There was incomplete data provided by the RN and MD. Overall, the two GCs spent 28.7 hours to set up the collaborative process, including organizational meetings and discussions, travel to the collaborative site to set up processes, educational training and observation (Table 1). The RN spent 34 hours on educational training, of which 28.6 hours was spent completing the ONS course and reading journal articles. Pre-implementation activities for the RN also included travel to the supportive site for observation, organizational meetings and tablet training, resulting in a total of 48.75 hours over a 3 -month time period. The MD did record her time spent on ONS activities, which was 10.3 hours. There was no change in provider knowledge test scores before and after implementation (data not shown).

The total time spent by the 2 genetic counselors (combined) is shown in Table 1. The total time spent inperson with the 8 patients ( 4 new and 4 follow-up) by a genetic counselor was 7.5 hours over the year. Implementation and organizational discussions continued throughout the year.

\section{Discussion}

This is the first report of a process that uses a collaborative approach to providing cancer genetic risk assessment at a small community-based hospital. Though this was a pilot, it demonstrates how a small facility can use resources at a larger tertiary hospital to provide quality services to their patients.

There were many benefits identified from this approach (Table 2). A collaborative approach allowed us to use local resources in an environment where patients were comfortable with providers and facility. It reduced the burden on the genetic counseling staff because many of the patients could be seen up front by the local $\mathrm{RN}$, requiring a follow-up appointment only, which is less time consuming. It provided direct access to a highly trained GC, which we argue would improve quality of care, although we do not yet have data to support that.

One of the primary benefits of using a collaborative approach was maximizing the unique skills of each professional involved (Figure 3). The oncology nurse has experience in oncology, patient education and psychosocial support skills. She was very familiar with the patients since she provides ongoing, local care and is familiar with local resources. It should be noted that, although we used an $\mathrm{RN}$ for our collaborative program, other nongenetics health care professionals may assume this role, including a nurse practitioner or physician assistant. The physician was often already involved providing care and could write orders for testing and make referrals for follow-up 
management. The physician also has relationships with local health care providers, lending authenticity and improving communication. The collaborating genetic counselor provides the expertise in genetics, patient education and psychosocial support skills. Additionally, the genetic counselor has experience in how health conditions may impact the entire family and is familiar with resources for hereditary cancer syndromes and research opportunities.

During this pilot period, this collaborative approach did improve identification of at-risk patients and increased access to hereditary cancer genetics services, though on a small scale. The total number of patients who accessed genetic counseling services from the target region was 4 times greater than the previous year (Figure 2). The number of patients served by our clinic at our main location may be an underestimate, since we searched our patient database by city, and there may be individuals who access care at the collaborative site but do not live within that city; however, we were consistent in using the same criteria to identify patients who came to the main clinic both before and during the study period.

Uptake was fairly high in this pilot, since 9 of 13 patients (69\%) who were offered risk assessment accepted the appointment. One reason for this very high acceptance rate could be that it was offered by a local, trusted health care practitioner where there was often already an established relationship, which is yet another potential advantage to using a collaborative approach.

Expanding this approach to a mammography unit in a smaller hospital could potentially identify unaffected individuals at-risk for hereditary cancer. At the collaborative site, approximately 25 mammograms are performed weekly. According to previous studies, $4 \%-8 \%$ of such a population may need risk assessment, depending on the method used, ${ }^{18}$ so a facility this size may have approximately an additional $75 \mathrm{pa}^{-}$ tients annually (range, 52-104) who would possibly benefit from risk assessment and/or genetic testing. Many of these would be initially offered BRCA1/2 testing, which could be achieved by the local staff, with back-up from the genetic 
TABLE 2 Lessons learned from a collaborative-approach pilot study

\begin{tabular}{ll} 
Benefits & Barriers \\
\hline - Improves identification of at-risk patients & - Up-front time investment \\
\hline - Increases access to genetic services & - Steep learning curve for non-genetics provider \\
\hline - Efficient use of cancer genetic counselor's time & - Staff motivation and desire for acquiring a new skill set \\
\hline - Utilizes local resources & - Administration buy-in \\
\hline - Patients tend to be comfortable with local providers and & \\
\hline local facility & - Reluctance of currently referring providers in adopting a new approach
\end{tabular}

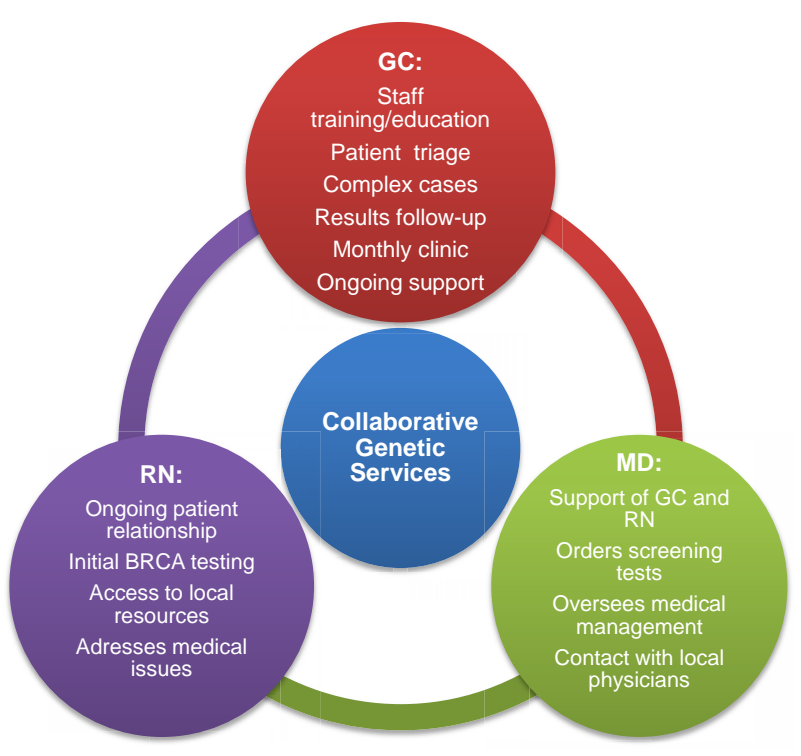

FIGURE 3 Roles in collaborative genetics services. Abbreviations: GC, genetic counselor; $M D$, physician; RN, registered nurse.

counselor, including follow-up and assessment for differentials and further testing. Some of these would be more complex cases that would require initial risk assessment from a board-certified genetic counselor.

There were some challenges in establishing our collaborative approach (Table 2). There was a steep learning curve for the nongenetics staff, and considerable investment was required upfront to train the health care providers involved. Motivation of selected staff members is a key factor, as this new skill set requires additional education and training. The time investment required of both the oncology nurse and genetic counselors were considerable. We had $1 \mathrm{RN}$ leave only 2 months after implementing the collaborative approach, which had a negative impact on the program. There is no way to predict when or if staff members will leave an institution, but fortunately we had trained 2 oncology nurses, so even after the departure of $1 \mathrm{RN}$, we still had a staff member with qualifications and training. The longer the staff members remain in their position, the better value on the investment for this type of collaborative approach. Additionally, we expect that as this established relationship remains in place, less time will be required of the collaborative genetic counselors.

Other unexpected issues that arose included some amount of resistance from administration of the local community hospital and currently referring providers. Although we had the support of the cancer center at the collaborative site, when higher-up administrators became aware of our program, they were concerned about the ethical implications of offering genetic testing. They had not been involved in the development of the program, so were not familiar with the goals and processes. We were able to overcome this concern by educating staff with a presentation of processes and goals to the ethics board. A takeaway lesson was to make hospital leadership aware of such a program prior to implementation, and not assume that because the oncology department is on-board, that hospital administrators will automatically accept a new program.

With regard to currently referring providers, there was some initial reluctance to accept a collaborative approach to genetic testing. Several radiation and medical oncologists from the main site also provide service to the collaborating site. They were familiar with the genetic counseling staff and had used our cancer genetic services in Indianapolis. These physicians expressed concern that nongenetics health care providers would be offering genetic testing to patients directly without seeing the genetic counselor first. To address this concern, we educated them about the process, how the oncology nursing staff had undergone training and the type of support that the collaborating genetic counselors were providing, both on a one-on-one basis and via tumor board presentation. It is important to note that there are many individuals at-risk for hereditary cancer and only a limited number of cancer 
genetic counselors. By having a GC involved in the process of genetic testing via training of nongenetics staff, this allows for better quality of care with increased access to services that otherwise would not likely be used. Another avenue that should be explored is telemedicine for those institutions that have access to necessary audiovisual equipment and methods for billing.

It was disappointing that provider knowledge test scores did not improve before and after implementation of the collaborative approach. There are a number of possible explanations for this observation. The physician did not have hands-on involvement in the process, as hers was a more supportive role, which may explain her unaltered test score. The RN did not take her test until 6 months after the study period ended, at which time the collaborative relationship was on hold pending a contract. This may demonstrate that continued exposure and use of skills is important to maintain knowledge. During the study period, the RN did express increased confidence, knowing she had support from the genetic counselors.

There are still some unknowns regarding this collaborative approach that warrant further investigation. We initially had intended to screen all new oncology patients who accessed radiation and medical oncology services at the collaborative site to determine the percentage of individuals who would be appropriate for cancer genetic counseling services. However, partly due to time constraints and staff turnover, the computerized survey was only offered to select individuals, determined by the RN with in-person questioning targeting certain diagnoses and/or age of onset. This prescreening resulted in a high percentage of individuals identified at increased risk for hereditary cancer. It also limited our ability to determine the percent of patients from a community cancer center who would be offered hereditary cancer risk assessment.

We had hoped to include data on downstream revenue to demonstrate financial feasibility of a collaborative program. However, with the small number of individuals who were identified at increased risk, we were not able to gather any meaningful data. We also had hoped to demonstrate a favorable impact on health care provider efficiency; but again, the data set was too small to evaluate. A significant amount of time was spent on travel by the genetic counselors to and from the remote site for the monthly visits. This highlights an opportunity for improvement in efficiency by providing genetic counseling via telegenetics for follow-up and triaged cases. There is still value in attending tumor board meetings, although this could be done via telephone or videoconferencing as well.
After the grant-funded study period ended, the collaborative site indicated interest in continuing the collaborative relationship to provide quality genetic counseling services to their patient population. A contract to provide services was initiated in October 2012, and we have a contract with a second community hospital in place using this collaborative approach as well. We will continue to monitor our time and resource use with the hopes of demonstrating widespread feasibility.

\section{Conclusion}

This pilot describes a workable model for patient identification and triage for hereditary cancer risk assessment and genetic counseling to improve access to boardcertified genetic counselors. We identified benefits and potential barriers to developing such a community-based collaborative approach, using remote involvement of board certified cancer genetic counselors from a larger off-site center. The collaborative approach can be applied to other health care facilities that do not currently have access to a board-certified genetic counselor as demand for genetic testing services is increasing for more common, adult-onset conditions. This may be especially important in light of the 2012 Commission on Cancer Standards that require cancer risk assessment, genetic counseling and testing services on site or by referral. ${ }^{13}$

\section{Acknowledgement}

Funding for this project was provided by the St. Vincent Foundation, Indianapolis, Indiana.

\section{References}

1. Sifri R, Myers R, Hyslop T, et al. Use of cancer susceptibility testing among primary care physicians. Clin Genet. 2003;64(4): 355-360.

2. Wideroff L, Freedman AN, Olson L, et al. Physician use of genetic testing for cancer susceptibility: results of a national survey. Cancer Epidemiol Biomarkers Prev. 2003;12(4):295-303.

3. Freedman AN, Wideroff L, Olson L, et al. US physicians' attitudes toward genetic testing for cancer susceptibility. Am J Med Genet A. 2003;120A(1):63-71.

4. Bottorff JL, Blaine S, Carroll JC, et al. The educational needs and professional roles of Canadian physicians and nurses regarding genetic testing and adult onset hereditary disease. Community Genet. 2005;8(2):80-87.

5. Sabatino SA, McCarthy EP, Phillips RS, Burns RB. Breast cancer risk assessment and management in primary care: provider attitudes, practices, and barriers. Cancer Detect Prev. 2007;31(5):375383.

6. Scheuner MT, Sieverding P, Shekelle PG. Delivery of genomic medicine for common chronic adult diseases: a systematic review. JAMA. 2008;299(11):1320-1334.

7. Carroll JC, Cappelli M, Miller F, et al. Genetic services for hereditary breast/ovarian and colorectal cancers - physicians' awareness, use and satisfaction. Community Genet. 2008;11(1):43-51.

8. Wideroff L, Vadaparampil ST, Greene MH, Taplin S, Olson L, Freedman AN. Hereditary breast/ovarian and colorectal cancer genetics knowledge in a national sample of US physicians. $J$ Med Genet. 2005;42(10):749-755. 


\section{Original Research}

9. Acheson LS, Stange KC, Zyzanski S. Clinical genetics issues encountered by family physicians. Genet Med. 2005;7(7):501508.

10. Riley BD, Culver JO, Skrzynia C, et al. Essential elements of genetic cancer risk assessment, counseling, and testing: updated recommendations of the National Society of Genetic Counselors. $J$ Genet Couns. 2012;21(2):151-161.

11. DeMarco TA, Smith KL, Nusbaum RH, Peshkin BN, Schwartz MD, Isaacs C. Practical aspects of delivering hereditary cancer risk counseling. Semin Oncol. 2007;34(5):369-378.

12. Brierley KL, Campfield D, Ducaine W, et al. Errors in delivery of cancer genetics services: implications for practice. Conn Med. 2010;74(7):413-423.

13. Greene FL, Dickson-Witmer D, Edge SB, et al; Commission on Cancer. Cancer Program Standards 2012: Ensuring PatientCentered Care. Chicago, IL: American College of Surgeons; 2012.

14. Bernhardt BA, Geller G, Doksum T, Metz SA. Evaluation of nurses and genetic counselors as providers of education about breast cancer susceptibility testing. Oncol Nurs Forum. 2000; 27(1):33-39.

15. Cohen SA, McIlvried D, Schnieders J. A collaborative approach to genetic testing: a community hospital's experience. J Genet Couns. 2009;18(6):530-533.

16. Daly M, et al. NCCN Guidelines Version 1.2012 Genetic/Familial High-Risk Assessment: Breast and Ovarian. 2012; Available from: nccn.org.

17. Nelson HD, Huffman LH, Fu R, Harris EL; U.S. Preventive Services Task Force. Genetic risk assessment and BRCA mutation testing for breast and ovarian cancer susceptibility: systematic evidence review for the U.S. Preventive Services Task Force. Ann Intern Med. 2005;143(5):362-379.

18. Palomaki GE, McClain MR, Steinort K, Sifri R, LoPresti L, Haddow JE. Screen-positive rates and agreement among six family history screening protocols for breast/ovarian cancer in a population-based cohort of 21- to 55-year-old women. Genet Med. 2006;8(3):161-168. 